\title{
Primary Ovarian Pregnancy: A Rare Entity.
}

\author{
Dr. Bangal V.B., Dr. Gitay Madhumita, Dr. S. Shashant
}

\section{Introduction}

Ectopic pregnancy is one of the most common gynaecological emergencies. The myriad of locations have a hierarchy of prevalence as well as associated mortality and morbidity.

Ovarian pregnancy is a rare entity which accounts for upto $3 \%$ of all ectopic pregnancies that is difficult to diagnose clinically.' It is the most common type of non-tubal ectopic pregnancy. ${ }^{2}$ Ovarian pregnancy is a diagnosis of exclusion. ART procedures have resulted in a rise of pregnancy outside the uterine cavity, yet ovarian pregnancy is still rare. We present a case of ruptured ovarian ectopic pregnancy which required an emergency laparotomy.

\section{Case Report}

A 22 year old Primigravida was referred from a rural hospital \& presented to the casualty of Pravara Hospital with history of one and a half month of amenorrhea and sudden onset severe pain in abdomen since 4 hours. Her gestational age as per her LMP was 7 weeks. Her previous menstrual cycles were regular, of 28-30 day length and normal flow. She had no history of contraceptive use and no history of drug intake. On examination, patient was pale, pulse rate was 110 beats per minute, blood pressure of 90/60 $\mathrm{mmHg}$. There was no history of fever or vomiting. Respiratory and cardiovascular system examination findings were normal. On per abdomen examination, guarding, rigidity and tenderness were present. Bowel sounds were audible. On per speculum examination, cervix appeared normal and vagina appeared pale. Per vaginal examination revealed tenderness in bilateral fornices. Her urinary beta hCG tested positive. Pelvic ultrasonography revealed empty uterine cavity, but with thickened endometrium. Collection of 750-1000 cc in pelvic cavity was seen and a mass of $7 \times 6 \times 6 \mathrm{~cm}$ was identified in the left adnexa. Hence, a preoperative diagnosis of a ruptured left tubal ruptured ectopic pregnancy was made and emergency laparotomy was performed. Intraoperatively, the uterus was of found to be normal in size and both fallopian tubes appeared normal. A ruptured left ovarian ectopic pregnancy was noted. Abdomen was filled with $1000 \mathrm{ml}$ of freshly clotted blood. Right ovary was normal. Left ovary was enlarged, and site of rupture was at the postero-lateral surface of the ovary. Active bleeding was noted from the site of rupture. There was persistent bleeding from the vessels at the rupture site despite plication and electrocauterization, therefore left sided salpingo-oopherectomy was performed and haemostasis was achieved. One unit of packed red blood cells was transfused. Post-operative period was uneventful and the patient recovered well. Specimen excised [Fig. 1(a)] was sent for histopathological study which revealed trophoblastic tissue in scan view [Fig. 1(b)] and high power (Fig. 2). Thus, the diagnosis of ruptured primary ovarian pregnancy was confirmed as all findings were consistent with Spiegelberg's criteria.

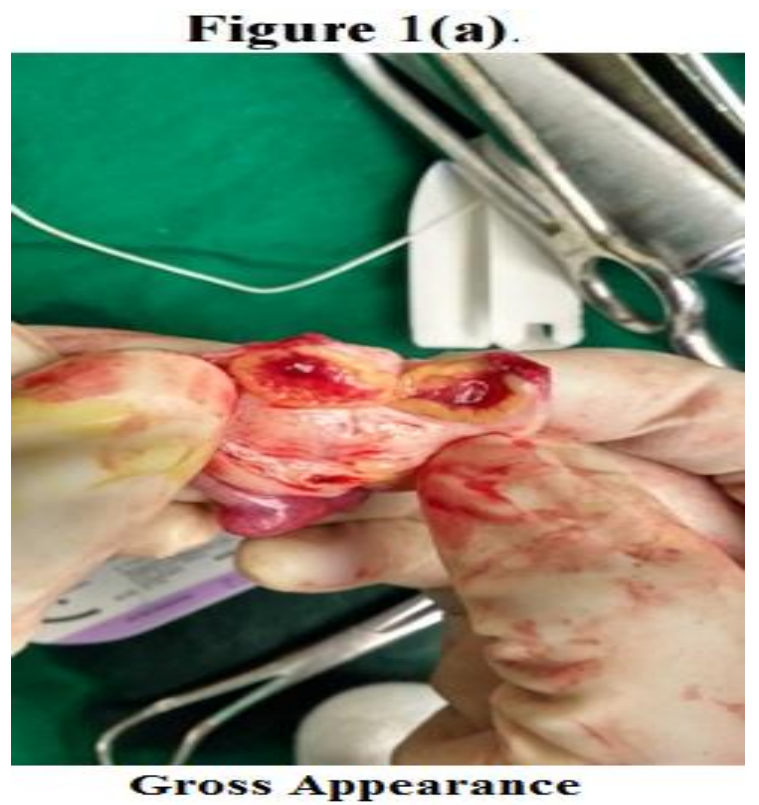




\section{Microscopic histopathology of trophoblast in the shelled out} Figure 1(b). gestational sac from the ovary

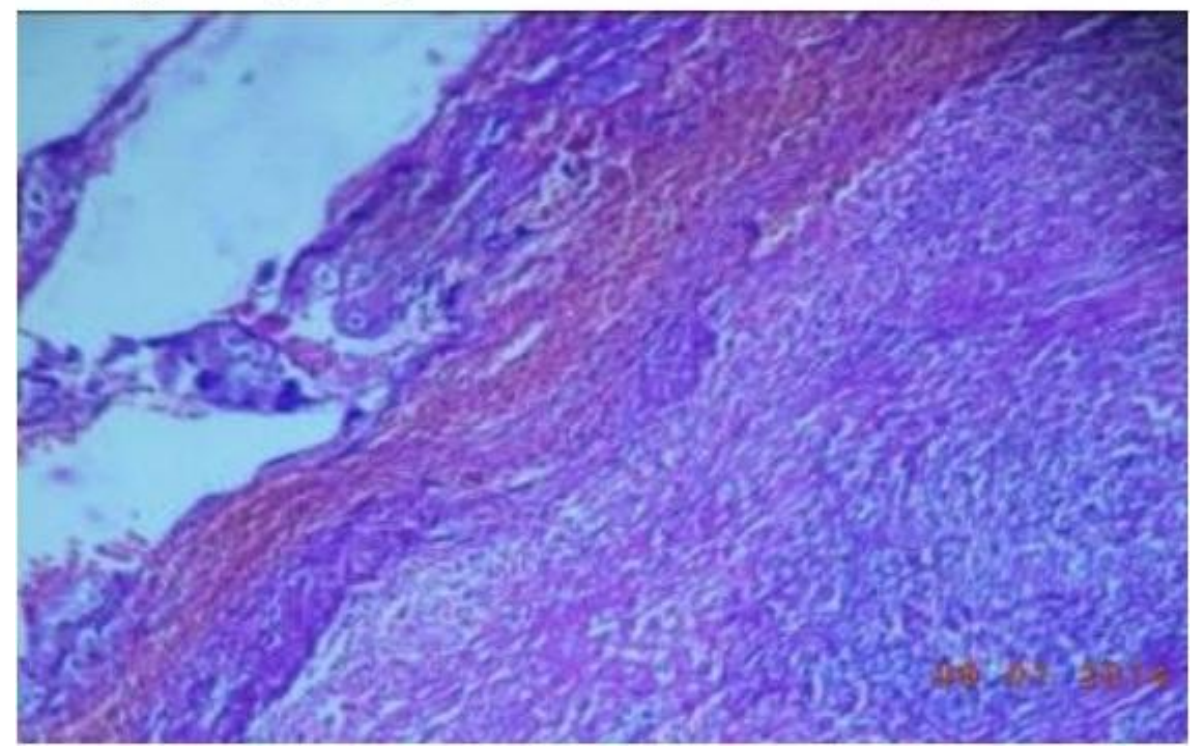

\section{Figure 2. High power view of a trophoblastic cell (centre)}

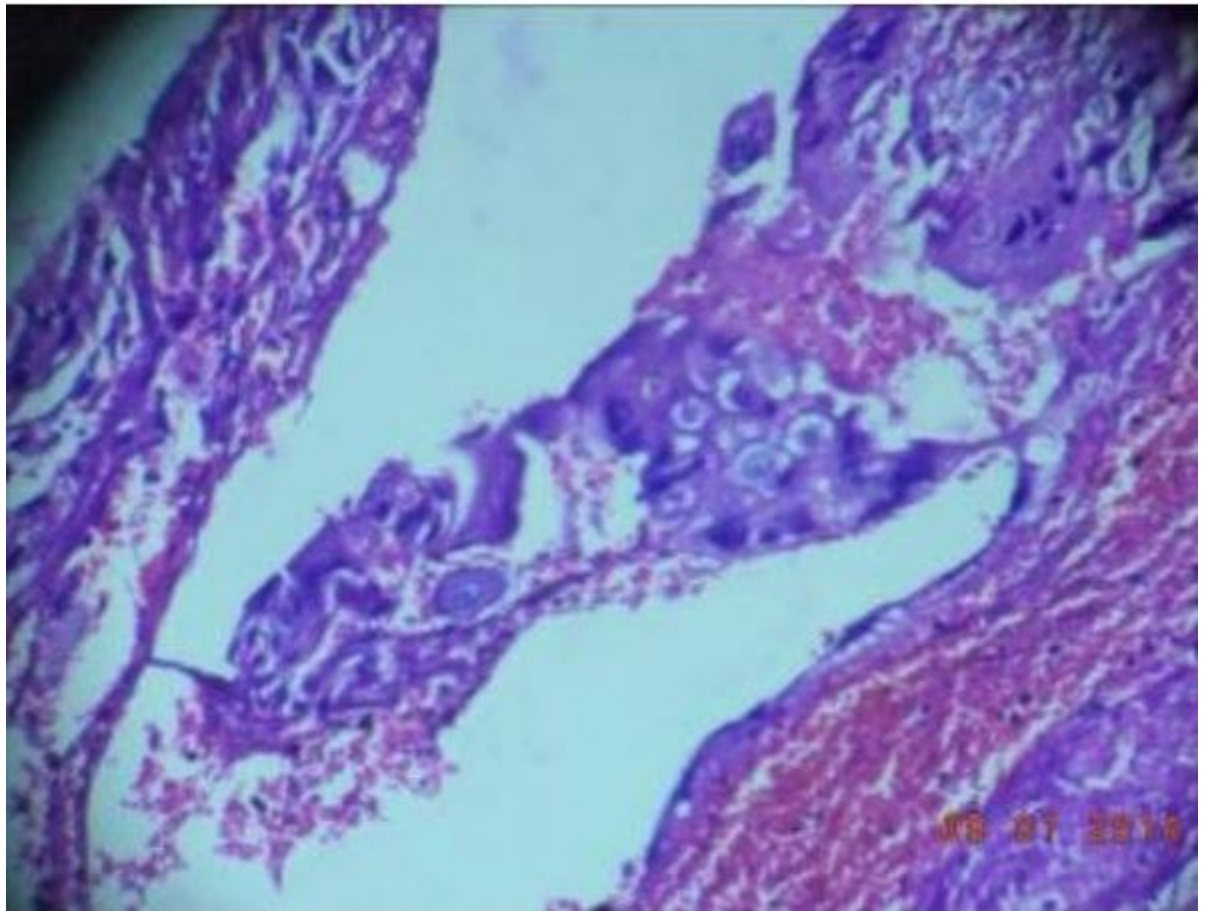

\section{Discussion}

Ectopic pregnancy is responsible for $10 \%$ of maternal mortality. Causes of ovarian pregnancy remain obscure. Primary ovarian pregnancy results when an oocyte is fertilized within the ovary, prior to ovulation OR following a primary implantation of fertilized ovum into the ovary after reverse migration from fallopian tube.

In a secondary ovarian pregnancy, there is a tubal abortion or rupture followed by secondary implantation of gestational sac on the surface of ovary. Primary ovarian pregnancy can be classified as intrafollicular or extrafollicular. ${ }^{3}$ In the intrafollicular type, the etiology is hormonal resulting in a trapped ovum inside the follicle. Another postulated cause is a thickened tunica albugenia of ovary. It may also result due to inefficient sweeping of fimbria ovarica across the surface of ovary resulting in ineffective ovum pick up. ${ }^{4}$ It is also associated with In-vitro fertilization/ ovulation induction and use of IUCDs. 
Spiegelberg suggested four criteria to distinguish a primary ovarian pregnancy from a distal tubal pregnancy which secondarily involved the ovary. These are:

1) The fallopian tube with its fimbriae should be intact and separate from the ovary.

2) The gestational sac should occupy the normal position of the ovary.

3) The gestational sac should be connected to the uterus by the ovarian ligament.

4) Histologically proven ovarian tissue should be located within the sac wall.

There is often a delay in the diagnosis of ovarian pregnancy as the gestational sac mimics: (a) corpus luteum, (b) hemorrhagic cyst and (c) chocolate cyst of ovary. It poses a significant diagnostic and therapeutic challenge and hence, carries a greater risk of maternal mortality than a tubal ampullary pregnancy.

With increasing use of ART procedures, clinicians should be equipped with skills to diagnose this evasive form of ectopic as early as possible. In our case, patient was having regular menstrual cycles of normal flow, and of 28 days duration. There was no history of infertility, sexually transmitted infections/PID, IUCD insertion, contraceptive pill use. Ovarian pregnancy occurs in fertile patients in contrary to tubal ectopic pregnancy which is commonly seen in patients with history of infertility. ${ }^{\mathbf{5}}$

Mean gestational age in literature is 45 days, which nearly corresponds to our patient's gestational age of 42 days. Patient usually presents with pain abdomen (100\%), vaginal bleeding (33\%) and hypovolemic shock $(8 \%) .{ }^{6}$ Our patient presented with abdominal pain and in hypovolemia. Diagnostic tests include the sensitive beta-hCG radioimmunoassay, culdocentesis and ultrasonography. TVS is a useful diagnostic tool. ${ }^{7} \mathrm{~A}$ hyperechoic white ring is seen at the location of the ovary which is more echogenic compared with surrounding ovarian tissue. A yolk sac or at times foetal parts may be visualized, but an embryonic pole is rarely seen. ${ }^{3}$ The corpus luteum has an anechoic texture and less wall echogenicity as compared to endometrium. An experienced sonologist is essential for an accurate ultrasound diagnosis.

Another diagnostic aid is laparoscopy, which has the advantage of permitting both diagnosis and treatment. One study has shown that early diagnosis of ovarian pregnancy with TVS allows for conservative management in the first trimester with methotrexate. ${ }^{\mathbf{}}$

Ovarian pregnancy usually (in over $90 \%$ of cases) ruptures in the first trimester. Our patient had a gestational age of 7 weeks. However, ovarian pregnancies are dangerous and prone for internal hemorrhage. Thus, when suspected, immediate laparotomy to arrest the site of hemorrhage is the life saving procedure. In the past, ovarian pregnancy had been treated by ipsilateral oophorectomy, but the trend has since shifted towards conservative surgery such as cystectomy or wedge resection performed at either laparotomy or laparoscopy. However, if the diagnosis is made later in the gestation, as in our patient, surgical treatment with ovarian resection or oophorectomy may be required. No case of recurrent ovarian ectopic has been reported which is in contrast to a $15 \%$ risk of recurrence of tubal ectopic pregnancy.

\section{Conclusion}

Ruptured primary ovarian pregnancy is rare type of ectopic pregnancy and can perplex the treating physician as it is difficult to diagnose clinically, radio logically as well as intraoperatively. The presence of adnexal mass on ultrasonography along with high index of clinical suspicion can lead to an early diagnosis and appropriate management of patient. Assisted reproduction procedures as well as other risk factors for ovarian pregnancy are on a rising trend, and clinicians should be well aware of this condition to diagnose it at the earliest.

\section{References}

[1]. Philippe E, Renaud R, Dellenbach P, Dreyfus J, Ritter J, Muhlstein C. Ovarian pregnancy. Apropos of 32 cases. J Gynecol Obstet Biol Reprod. 1987;16(7):901-8

[2]. Bouyer J, Coste J, Fernandez H,et al. Sites of ectopic pregnancy : a 10 year population-based study of 1800 cases. Hum Reprod 2002;17:3224-3230

[3]. Kaur, H., Shashikala, T., Bharath, M., Shetty, N., Rao, K.A. (2011). Ovarian ectopic pregnancy following assistive reproductive techniques: A rare entity. IJIFM., 2: 3739.

[4]. Zaidi, M.T, Ansari, M.S., Kirmani, F., Khan, A.A. (2012). A histoarchitectural study of early human ectopic pregnancy. Biomed. Res., 23 (1): 5156.

[5]. Seinera P, Gregorio A, Arisio R, Decko A, Crana F. Ovarian pregnancy and operative laparoscopy: Report of eight cases. Hum Reprod. 1998;12(3):608- 10.

[6]. Raziel A, Schachter M, Mordechai E, Friedler S, Panski M, Ron-El R. Ovarian pregnancy - a 12-year experience of 19 cases in one institution. Eur J Obstet Gynecol Reprod Biol. 2004;114(1):92-6.

[7]. Royal College of Obstetricians and Gynaecologists. Management of tubal pregnancy. Green Top Guidelines. London: RCOG; Guideline No. 21; May 2004

[8]. Chelmow, D., Gates, E., Penzias, A.S. (1994). Laparoscopic diagnosis and methotrexate treatment of an ovarian pregnancy: a case report. Fertil. Steril., 62: 87988 